\title{
Robustness of Delta Operator Based Cellular Neural Networks
}

\author{
BAHRAM MIRZAI ${ }^{1}$, HARI C. REDDY ${ }^{2,3}$ AND GEORGE S. MOSCHYTZ ${ }^{1}$ \\ ${ }^{1}$ Signal and Information Processing Laboratory, Swiss Federal Institute of Technology, Zurich, Switzerland \\ ${ }^{2}$ Department of Electrical Engineering, California State University, Long Beach, CA, USA \\ ${ }^{3}$ Department of Electrical and Computer Engineering, University of California, Irvine, CA, USA \\ mirzaio1si.ee.ethz.ch \\ hreddyoengr. csulb.edu
}

\begin{abstract}
The delta operator approach to continuous-time cellular neural networks (CT-CNNs) is investigated in terms of a robust realization. It is shown that earlier results concerning the robustness of CT. CNNs can be obtained as a limiting case of this approach, while at the same time, this allows us to formulate robustness considerations for discrete-time CNNs.
\end{abstract}

\section{Introduction}

Continuous-time cellular neural networks (CT-CNN) were first introduced in [1]. Primary applications were concerned with image processing tasks, e.g., $[1,2]$. Other areas of application, such as signal processing [3] and pattern recognition [4], have also been successfully considered. Although the basic equation describing the dynamics of each unit (cell) can be given in arbitrary dimensions, due to applications and implementation aspects, CNNs in two dimension have been particularly the subject of active research.

In this case, the dynamics of each unit is governed by

$$
\begin{aligned}
C \frac{d x_{i j}(t)}{d t} & =-\frac{1}{R} x_{i j}(t)+\sum_{m n \in \mathcal{N}_{i j}} a_{i j, m n} \operatorname{sat}\left(x_{m n}(t)\right) \\
& +\sum_{m n \in \mathcal{N}_{i j}} b_{i j, m n} u_{m n}+I,
\end{aligned}
$$

where sat $(\cdot)$ is the piece-wise linear non-linearity given by

$$
\operatorname{sat}(x)=\frac{1}{2}\{|x+1|-|x-1|\} .
$$

The inputs and outputs of CNNs are defined to be $u_{i j}$ and sat $\left(x_{i j}\right)$, respectively. Spatially invariant $\mathrm{CNNs}$ are specified by a template set $A, B$, containing $a_{i j}$ and $b_{i j}$, respectively, and a bias $I$. The operation of a CNN consists in appropriately choosing the template values $A, B$ and the bias $I$ such that for given initial values $x_{i j}(0)$ some desired image is obtained as the output of the system (1) at a stable equilibrium.

Along the implementation of CT-CNNs $[5,6]$, discretetime CNNs were considered in terms of implementation as sampled-data systems [7]. Analogously to (1), the basic dynamic equation, in this case, is given by

$$
x_{i j}[k+1]=\sum_{m n \in \mathcal{N}_{i j}} a_{i j, m n} \mathrm{f}\left(x_{m n}[k]\right)+\sum_{m n \in \mathcal{K}_{i j}} b_{i j, m n} u_{k l}+I
$$

where the non-linearity $f(\cdot)$ can be the hard limiting function $\operatorname{sgn}(\cdot)$, as introduced in [8], or the saturation function sat $(\cdot)$. Henceforth, we denote the former case by SGNCNNs and the latter by SAT-CNNs. As shown below, for sampled data systems, the advantage of the saturation nonlinearity to the hard limiter lies in the fact that in the former case the state of each cell depends continuously on the dynamic parameters, i.e., on the $A$-template.

In this paper we introduce the delta operator based approach to CNNs ( $\delta$-CNNs). As it is shown, this provides us with a unifying frame work for CT-CNNs and SAT-CNNs. By doing so, some of the results concerning the robustness of template sets, previously proved for CT-CNNs [9], can be formulated for $\delta$-CNNs. The respective continuous-time results are then obtained as a limiting case of the delta operator approach. At the same time, we can apply these results to SAT-CNNs.

In Sec. 2 we introduce $\delta$-CNNs. Sec. 3 compares SATCNNs and SGN-CNNs in terms of their continuous dependency on the dynamic parameters and deals with the robustness of $\delta$-CNNs. Sec. 4 provides the simulation results. We conclude with Sec. 5 .

\section{Delta operator based CNNs}

Given a continuous-time signal $x(t)$, we can obtain a discrete-time sequence $x\left(k T_{s}\right)$ by sampling $x(t)$ at a sampling rate of $T_{s}$. The sampled signal is denoted by $x[k]$ for $k \in \mathbb{Z}$.

The delta operator is defined on the set of causal sequences by [10]

$$
\delta x[k]=\frac{x[k+1]-x[k]}{T_{s}} .
$$

Clearly, for $x[k]=x\left(k T_{s}\right)$, i.e., resulting from sampling a continuous-time signal at period $T_{s}$, the delta operator is 
an approximation of its derivative. In the limiting case of $T_{s} \rightarrow 0$, we obtain

$$
\lim _{T_{s} \rightarrow 0} \delta x[k]=\left.\dot{x}(t)\right|_{t=k T_{s}} .
$$

For an inherently discrete system, as noted in [11], $T_{s}$ can be considered as an extra design parameter that can be used to achieve better performance for the underlying digital system.

The dynamic equation of a delta operator based discretetime $\mathrm{CNN}$ is defined as follows:

$$
\begin{aligned}
C \delta x_{i j}[k] & =-\frac{1}{R} x_{i j}[k]+\sum_{m n \in \mathcal{N}_{i j}} a_{i j, m n} \operatorname{sat}\left(x_{m n}[k]\right) \\
+ & \sum_{m n \in \mathcal{N}_{i j}} b_{i j, m n} u_{m n}+1 .
\end{aligned}
$$

By expanding (4) according to (3), we obtain

$$
\begin{gathered}
x_{i j}[k+1]=\left(1-\frac{T_{s}}{\tau}\right) x_{i j}[k]+\sum_{m n \in \mathcal{N}_{i j}} \frac{T_{s}}{C} a_{i j, m n} \\
\times \operatorname{sat}\left(x_{k l}[k]\right)+\sum_{m n \in \mathcal{N}_{i j}} \frac{T_{s}}{C} b_{i j, m n} u_{m n}+\frac{T_{s}}{C} I,
\end{gathered}
$$

where $\tau=R C$ defines the internal time scale of the analog system. If (5) is considered in conjunction with (1), then the sampling period $T_{s}$ has to satisfy $T_{s} \leq \tau$ in order to avoid aliasing effects. On the other hand, if (5) is considered by itself, because it does not contain any continuous-time integration, i.e., capacitance, there is no concept of an internal time and, hence, no restrictions of this kind on $T_{s}$. In the following, we consider (5) as an inherently digital system and normalize both $R$ and $C$ to unity. Clearly, CT-CNNs and SAT-CNNs can be obtained as limiting cases of $\delta$-CNNs, namely as $T_{s} \rightarrow 0$ and $T_{s} \rightarrow 1$, respectively.

\section{Robustness of $\delta$-CNNs}

In this section, we show that for discrete-time CNNs the use of piece-wise linear non-linearity provides us with a continuous dependency of the state trajectory on the $A$-template, whereas this is not the case for the hard limiting non-linearity. Further, we derive for $\delta$-CNNs necessary and sufficient bounds on the perturbations such that within these bounds the desired operation is correctly performed. The continuity property proves to be crucial to a design of robust templates. In the absence of a continuous dependency smallest perturbations chosen within the range suggested by the necessary condition may render a correct operation invalid.

We first introduce some notation and state a lemma. Let $\|\cdot\|_{\infty}$ denote the max-norm defined by $\|\mathbf{x}\|_{\infty}=\max _{i}\left|x_{i}\right|$. The corresponding induced matrix norm, denoted again by $\|\cdot\|_{\infty}$, is

$$
\|\mathbf{M}\|_{\infty}=\max _{\|\mathbf{x}\|_{\infty}=1}\|\mathbf{M} \mathbf{x}\|_{\infty}=\max _{i} \sum_{j}\left|M_{i j}\right| .
$$

The following estimate holds for the induced matrix norm: $\|\mathbf{M x}\|_{\infty} \leq\|\mathbf{M}\|_{\infty}\|\mathbf{x}\|_{\infty}$. By using vector notation, the dynamic equations (5) can be written as

$$
\begin{aligned}
& \mathbf{x}[k+1]=\left(1-T_{s}\right) \mathbf{x}[k]+T_{s} \mathbf{A} \operatorname{sat}(\mathbf{x}[k]) \\
& \quad+T_{s} \mathbf{B} \mathbf{u}+T_{s} \mathbf{i}
\end{aligned}
$$

Note that in (6) we assumed zero boundary values. Henceforth, this will be the case unless it is stated to be otherwise. In the following, the entries of $\mathbf{A}$ and $\mathbf{B}$ are denoted by $A_{i j}$ and $B_{i j}$, respectively. For $\mathbf{A}$, as given in (6), we obtain

$$
\|\mathbf{A}\|_{\infty}=\max _{i} \sum_{j}\left|A_{i j}\right|=\sum_{i j}\left|a_{i j}\right| \text {. }
$$

Lemma: Suppose $h, g \in \mathbb{R}^{+}$and $\theta_{n} \geq 0$ be such that

$$
\theta_{n} \leq(1+h) \theta_{n-1}+g \text {, }
$$

then

$$
\theta_{n} \leq(1+h)^{n} \theta_{0}+\sum_{i=0}^{n-1}(1+h)^{i} g
$$

The proof can be readily obtained, e.g., by induction. In particular, for $\theta_{0}=0$ we obtain

$$
\theta_{n} \leq \frac{(1+h)^{n}-1}{h} g \text {. }
$$

The continuity property is given by the following theorem. Theorem: The state vector $\mathbf{x}[k]$, as determined by (6), depends continuously on $\mathbf{A}$, i.e., for every $\varepsilon>0$ there is $\eta>0$ such that if $\|\mathbf{A}-\tilde{\mathbf{A}}\|_{\infty} \leq \eta$ then $\|\mathbf{x}[k]-\tilde{\mathbf{x}}[k]\|_{\infty} \leq \varepsilon$.

Proof: Let $\tilde{\mathbf{A}}=\mathbf{A}+\Delta \mathbf{A}$. From (6) the following estimate can be made

$$
\begin{aligned}
& \|\mathbf{x}[k+1]-\tilde{\mathbf{x}}[k+1]\|_{\infty} \leq\left|1-T_{s}\right|\|\mathbf{x}[k]-\tilde{\mathbf{x}}[k]\|_{\infty} \\
& \quad+T_{s}\|\mathbf{A}\|_{\infty}\|\operatorname{sat}(\mathbf{x}[k])-\operatorname{sat}(\tilde{\mathbf{x}}[k])\|_{\infty} \\
& \quad+T_{s}\|\Delta \mathbf{A}\|_{\infty}\|\operatorname{sat}(\tilde{\mathbf{x}}[k])\|_{\infty},
\end{aligned}
$$

and from (8) it follows

$$
\begin{aligned}
& \|\mathbf{x}[k+1]-\tilde{\mathbf{x}}[k+1]\|_{\infty} \leq\left(1+T_{s}+T_{s}\|\mathbf{A}\|_{\infty}\right) \\
& \quad \times\|\mathbf{x}[k]-\tilde{\mathbf{x}}[k]\|_{\infty}+T_{s}\left\|_{1} \Delta \mathbf{A}\right\|_{\infty}
\end{aligned}
$$

By applying the lemma to (9) with $\theta_{k}=\|\mathbf{x}[k]-\tilde{\mathbf{x}}[k]\|_{\infty}$ $h=T_{s}+T_{s}\|\mathbf{A}\|_{\infty}$ and $g=T_{s}\|\Delta \mathbf{A}\|_{\infty}$, from (7) we obtain

$$
\|\mathbf{x}[k]-\tilde{\mathbf{x}}[k]\|_{\infty} \leq \frac{\left(1+T_{s}+T_{s}\|\mathbf{A}\|_{\infty}\right)^{k}-1}{1+\|\mathbf{A}\|_{\infty}}\|\Delta \mathbf{A}\|_{\infty} .
$$

By choosing

$$
\eta \leq \frac{1+\|\mathbf{A}\|_{\infty}}{\left(1+T_{s}+T_{s}\|\mathbf{A}\|_{\infty}\right)^{k}-1} \varepsilon,
$$

from (10) follows the continuity. This completes the proof of the theorem. 
A similar statement cannot be made if the non-linearity in (6) is replaced by the hard limiting function. In the case of the $\operatorname{sgn}(\cdot)$ function (9) would correspond to

$$
\begin{aligned}
& \|\mathbf{x}[k+1]-\tilde{\mathbf{x}}[k+1]\|_{\infty} \leq 2\left(1+T_{s}+T_{s}\|\mathbf{A}\|_{\infty}\right) \\
& \quad+T_{s}\|\Delta \mathbf{A}\|_{\infty},
\end{aligned}
$$

which precludes any continuity conclusion.

We proceed by deriving necessary and sufficient bounds on the perturbation parameters. Equation (6) provides us with the starting point of the robustness analysis. Here we closely follow the approach introduced in [9] for CT-CNNs. Consider a template set $A, B$ and $I$, that performs some specific task and assumes its equilibrium point in a saturation region of the state space. Let $\Delta A, \Delta B$ and $\Delta I$ be a perturbation of the nominal values. The goal of the robustness study is to give bounds on the perturbation parameters such that the specific task under consideration is still correctly performed by the perturbed set, namely $A+\Delta A, B+\Delta B$ and $I+\Delta I$.

A necessary condition for the perturbed system to converge at the same saturation region as the unperturbed system is obtained by exploring the output invariance, i.e.,

$$
\operatorname{sat}\left(x_{p}^{*}\right)=\operatorname{sat}\left(x^{*}\right),
$$

where $*$ denotes the steady state, i.e., $\mathbf{x}^{*}[k+1]=\mathbf{x}^{*}[k]$ and $\mathbf{x}_{p}$ is the state vector of the perturbed system given by

$$
\begin{aligned}
& \mathbf{x}_{p}[k+1]=\left(1-T_{s}\right) \mathbf{x}_{p}[k]+T_{s}(\mathbf{A}+\Delta \mathbf{A}) \operatorname{sat}\left(\mathbf{x}_{p}[k]\right) \\
& \quad+T_{s}(\mathbf{B}+\Delta \mathbf{B}) \mathbf{u}+T_{s}(\mathbf{i}+\Delta \mathbf{i}) .
\end{aligned}
$$

Assuming (11), from (12) follows at the steady state

$$
\mathbf{x}_{p}^{*}=\mathbf{x}^{*}+\Delta \mathbf{A} \operatorname{sat}\left(\mathbf{x}^{*}\right)+\Delta \mathbf{B u}+\Delta \mathbf{i} .
$$

The last equation relates the equilibria of the perturbed and unperturbed systems, assuming that they are in the same saturation region. By inserting (13) back into (11) we obtain, as a necessary condition, the following bounds on the perturbation parameters

$$
\Delta_{\text {total }}<\min _{k, \mathbf{1}_{ \pm}}\left|\left(\mathbf{A} \mathbf{1}_{ \pm}+\mathrm{Bu}+\mathbf{i}\right)_{k}\right|-1,
$$

where $\Delta_{\text {total }}=\sum_{i j}\left(\left|\Delta a_{i j}\right|+\left|\Delta b_{i j}\right|\right)+|\Delta I|$ and $\mathbf{1}_{ \pm}$is any bipolar vector that can occur as an output of the template set $A, B$ and $l$. The minimum in (14) is taken over all cells, i.e., components of the state vector. Note that since (13) does not contain any dependency on the sampling period $T_{s}$, the necessary condition (14) does not either. In particular, CT-CNNs and $\delta$-CNNs do not differ in this respect [9]. Moreover, because the state vector, by assumption, converges in a saturation region and (14) is derived by considering the steady state, we will also get the same bound for $\Delta_{\text {lotal }}$ as in (14) if we replace sat $(\cdot)$ by $\operatorname{sgn}(\cdot)$.
We proceed by estimating the deviation in the state vector caused by the perturbation

$$
\begin{aligned}
& \mathbf{x}_{p}[k+1]-\mathbf{x}[k+1]=\left(1-T_{s}\right)\left(\mathbf{x}_{p}[k]-\mathbf{x}[k]\right. \\
& \quad+T_{s} \mathbf{A}\left(\operatorname{sat}\left(\mathbf{x}_{p}[k]\right)-\operatorname{sat}(\mathbf{x}(k))\right)+T_{s} \Delta \mathbf{A} \operatorname{sat}\left(\mathbf{x}_{p}\right) \\
& \quad+T_{s} \Delta \mathbf{B u}+T_{s} \Delta \mathbf{i},
\end{aligned}
$$

Assuming all inputs to be in magnitude less than unity, by applying the max-norm $\|\cdot\|_{\infty}$ and the lemma to (15) we obtain

$$
\left\|\mathbf{x}_{p}[k]-\mathbf{x}[k]\right\|_{\infty} \leq \frac{\left(1+T_{s}+T_{s}\|\mathbf{A}\|_{\infty}\right)^{k}-1}{1+\|\mathbf{A}\|_{\infty}} \Delta_{\text {tutal }} .
$$

As a sufficient condition, we require the deviation due to perturbation be such that

$$
\left\|\mathbf{x}_{p}[k]-\mathbf{x}[k]\right\|_{\infty}<\min _{i}\left|x_{i}^{*}\right|-1 \quad \forall k>k^{*},
$$

where $k^{*}$ is the smallest time for which both the perturbed and unperturbed system enter their steady state. Equation (17) in conjunction with (16) implies now the following sufficient bound on $k^{*}$ to get the correct output in the presence of the total perturbation $\Delta_{\text {total }}$

$k^{*}<\frac{\log \left(\left(1+\|\mathbf{A}\|_{\infty}\right)\left(\min _{k}\left|x_{k}^{*}\right|-1\right)+\Delta_{\text {total }}\right)-\log \left(\Delta_{\text {total }}\right)}{\log \left(1+T_{s}\left(1+\|\mathbf{A}\|_{\infty}\right)\right)}$.

We make the following remarks concerning (18):

i) For $\Delta_{\text {total }}=0$, i.e., if the perturbation is turned off, the upper bound in (18) tends to infinity. This is consistent with the initial assumption that the unperturbed system converges to some stable equilibrium.

ii) If the unperturbed system is already non-robust, i.e., $\min _{i}\left|x_{i}^{*}\right|-1=0$, then the upper bound in (18) becomes zero, which again is a consistency indication.

iii) The bound imposed on $k^{*}$ in (18) reduces to the corresponding bound in the continuous-time case [9] as $T_{s}$ tends to zero. Due to $\lim _{T_{s} \rightarrow 0} \frac{\log \left(1+T_{s} a\right)}{T_{s}}=a$ and $T_{s} k \rightarrow t$, we obtain from (18)

$$
t^{*}<\frac{\log \left(\left(1+\|\mathbf{A}\|_{\infty}\right)\left(\min _{k}\left|x_{k}^{*}\right|-1\right)+\Delta_{\text {total }}\right)-\log \left(\Delta_{\text {total }}\right)}{1+\|\mathbf{A}\|_{\infty}},
$$

where $t^{*}$ is the continuous analogue of $k^{*}$, i.e., the time required for both the perturbed and unperturbed systems to settle.

\section{Simulation results}

Simulations are performed to compare $\delta$-CNNs and SGNCNNs in terms of template perturbations. For simplicity, we consider applications for which $B=0$. In fact, from the dynamic point of view this is the interesting case. Although in the analysis of the previous sections the boundary was assumed to be the fixed value of zero, for simulations we set the boundary to the fixed value of -1 . This choice allows 
us to avoid the influence of the boundary on the sensitivity of the templates to be considered [9].

For completeness, we list the simulated systems including the boundary term and assuming $B=0$ :

$$
\begin{aligned}
\dot{\mathbf{x}}(t) & =-\mathbf{x}(t)+\mathbf{A} \operatorname{sat}(\mathbf{x}(t))+\mathbf{i}+\partial \\
\mathbf{x}[k+1] & =\left(1-T_{s}\right) \mathbf{x}[k]+T_{s} \mathbf{A} \operatorname{sat}(\mathbf{x}[k])+T_{s} \mathbf{i}+T_{s} \partial \\
\mathbf{x}[k+1] & =\mathbf{A} \operatorname{sgn}(\mathbf{x}[k])+\mathbf{i}+\partial,
\end{aligned}
$$

where $\partial$ denotes the contribution of the boundary cells. In the presence of non-zero boundary values, the necessary condition in (14) becomes

$$
\sum_{i j}\left|\Delta a_{i j}\right|+|\Delta I|<\min _{k, \mathbf{1}_{ \pm}}\left|\left(\mathbf{A} \mathbf{1}_{ \pm}+\mathbf{i}+\partial\right)_{k}\right|-1 .
$$

where, in view of simulations, we again assumed $B=0$.

As a first example, we consider the following template set: $A=\left[\begin{array}{lll}0 & 2 & q\end{array}\right], I=1$. For $q=1$, this corresponds to a shadowing template of the CT-CNN (20) with $\mathbf{x}(0)$ being initialized with the image to be processed. Assuming we consider only perturbations with respect to $q$, from (23) it follows that $|\Delta q|<\min _{k, 1_{ \pm}}\left|\left(\mathbf{A} 1_{ \pm}+\mathbf{i}+\partial\right)_{k}\right|-1=1$. Simulations of (21) with $T_{s}=0.01,1$ and $q=1$ show that (21) also performs the shadowing task with $|\Delta q|<1$. Furthermore, simulations of (21) with $T_{s}=0.01,1$ and $q$ being randomly chosen in $[0.1,1.9]$ lead still to the correct output. However, although the SGN-CNN (22) for $q=1$ gives the desired output with $\min _{k, 1_{ \pm}}\left|\left(\mathbf{A} 1_{ \pm}+\mathbf{i}+\partial\right)_{k}\right|-1=1$, but for any perturbation of the nominal value $q=1$ result ing in a $q<1$ it fails to converge to the correct output. This demonstrates the non-continuous dependency of the state vector on the $A$-template in the case of hard limiting nonlinearity.

Next, we consider the template set $A=\left[\begin{array}{lll}1 & 2 & q\end{array}\right], I=0$. For $q=-1$ this is a connected component detector (CCD) for (20). From (23) can be shown that the total tolerable perturbation for $q=-1$ is upper bounded by $|q|<1$. Simulations performed with (20) show, in fact, a robustness of the same amount [12]. The $\delta$-CNN (21) with $T_{s}=0.01,1$ and $q=-1$ behaves also like a CCD. In fact, the correct operation is guaranteed for any perturbation of $q$ satisfying $|\Delta q|<1$. On the other hand, the SGN-CNN (22) fails to converge to the correct output for $q=-1$. Hence, a template set that works for CT-CNNs may fail if used for SGN-CNNs, however, it will apply to $\delta$-CNNs. Further, for $q=-1.01$, (22) performs CCD correctly with $\min _{k, 1_{ \pm}}\left|\left(\mathbf{A} 1_{ \pm}+\mathbf{i}+\partial\right)_{k}\right|-1=0.99$, showing the non-continuous behavior of the SGN-CNNs.

\section{Conclusions}

A delta operator based approach to $\mathrm{CNNs}$ was introduced. A comparison of the delta discrete-time CNNs with the conventional ones utilizing a hard limiting function was made. It turns out that $\delta$-CNNs resembles CT-CNNs both in dynamics and in robustness, whereas SGN-CNNs may differ abruptly. In particular, the absence of a continuous dependency of SGN-CNNs on the $A$-template may render a robust design of them hard. Robust operation is desired if, e.g., the data to be processed is subject to noise.

\section{References}

[1] L. O. Chua and L. Yang, "Cellular Neural Networks: Theory," IEEE Transactions on Circuits and Systems, vol. 35, pp. 1257-1272, Oct. 1988.

[2] T. Yang, L.-B. Yang, C. W. Wu, and L. O. Chua, "Fuzzy Cellular Neural Networks: Applications," in IEEE International Workshop on Cellular Neural Networks and their Applications, (Seville), pp. 225-230, June 1996.

[3] J. A. Osuna, The Recognition of Acoustical Alarm Signals with Cellular Neural Networks. PhD thesis, Diss. ETH Zurich, Nr. 11058, Swiss Federal Institute of Technology, ETH-Center, CH-8092 Zurich, Switzerland, 1995. ISBN 3-89191-953-0.

[4] P. Thiran, K. R. Crounse, L. O. Chua, and M. Hasler, "Pattern Formation Properties of Autonomous Cellular Neural Networks," IEEE Transactions on Circuits and Systems-I, vol. 42, pp. 757-774, Oct. 1995.

[5] D. Lím and G. S. Moschytz, "A Programmable, Modular CNN Cell," in IEEE International Workshop on Cellular Neural Networks and their Applications, (Rome), pp. 79-84, Dec. 1994.

[6] S. Espejo, R. Domínguez-Castro, and A. RodríguezVázquez, "A Realization of a CNN Universal Chip in CMOS Technology," in IEEE International Symposium On Circuits And Systems, vol. 3, (Seattle), pp. 657-659, May 1995.

[7] H. Harrer and J. A. Nossek, "An analog implementation of discrete-time CNNs," IEEE Transactions on Neural Networks, vol. 3, 1992.

[8] H. Harrer and J. A. Nossek, "Discrete-time cellular neural networks," International Journal of Circuit Theory and Applications, vol. 20, Sept. 1992.

[9] B. Mirzai and G. S. Moschytz, "The Influence of the Boundary Conditions on the Robustness of a CNN," July 1996. submitted for publication.

[10] G. C. Goodwin, R. H. Middleton, and H. V. Poor, "High-speed Digital Signal Processing and Control," Proceedings of the IEEE, vol. 80, pp. 240-259, Feb. 1992.

[11] K. Premaratne and P. H. Bauer, "Delta-operator Formulated Disctrete-time Systems," in IEEE International Symposium On Circuits And Systems, vol. 2, (Atlanta), pp. 528-531, May 1996.

[12] B. Mirzai, D. Lím, and G. S. Moschytz, "Robust CNN Templates: Theory and Simulations," in IEEE International Workshop on Cellular Neural Networks and their Applications, (Seville), pp. 393-398, June 1996. 